\title{
Roboter-Tiere mit künstlicher Intelligenz (KI) - verheißungsvolles Experiment oder technisches K. O.?
}

\section{Christina Potschka}

Weltweit entwickeln Forscher eine Vielzahl von Roboter-Tieren, die nicht ausschließlich Fähigkeiten von natürlich vorkommenden Tieren besitzen, sondern diese in Zukunft übertreffen sollen. Der technische Fortschritt und die damit einhergehenden steigenden Fähigkeiten von menschlich konstruierten, künstlich intelligenten Tieren führen zu einem vermehrten Einsatz von Roboter-Tieren sowohl in der menschlichen wie auch in der tierischen Lebenswelt.

Optisch gleichen Roboter-Tiere natürlich entwickelten Tieren. Wie sich Roboter-Tiere im Vergleich zu natürlich entwickelten Tieren unterscheiden und weshalb sie Teil des tierethischen Diskurses sein sollen, wird im Folgenden dargelegt. Hierzu wird zunächst geklärt, was unter natürlich vorkommenden Tieren zu verstehen ist und welche Arten von RoboterTieren es bisher gibt. Anschließend werden Eigenschaften und Fähigkeiten von natürlich vorkommenden Tieren herausgearbeitet und mit denen von Roboter-Tieren verglichen. In einem dritten Schritt wird mittels einer Studien- und Wildtierdokumentationsanalyse überprüft, inwieweit Menschen und Tiere Roboter-Tiere akzeptieren, mit ihnen interagieren und arteigene Eigenschaften auf sie attribuieren. Die Analyse wird zeigen, dass es, ähnlich wie bei Menschen den Anthropomorphismus, im Tierreich den Animal-Morphismus, kurz "Animorphismus“, gibt. Abschließend werden zwei tierethische Ansätze herangezogen, um aufzuzeigen, inwiefern Roboter-Tieren ein inhärenter Wert und moralischer Status zusteht.

\section{1. Überblick: Natürlich entwickelte Tiere und Roboter-Tiere}

\section{Natürlich entwickelte Tiere}

Ein natürlich entwickeltes Tier ist ein „mit Sinnes- und Atmungsorganen ausgestattetes, sich von anderen tierischen oder pflanzlichen Organismen ernährendes, in der Regel frei bewegliches Lebewesen, das nicht (oder weniger stark als der Mensch) zu logischem Denken und zum Sprechen befähigt ist“" (Dudenredaktion 2019). 
Eine systematische Einteilung des Tierreiches kann auf unterschiedliche Art und Weise erfolgen. Natürlich entwickelte Tiere lassen sich in Einzeller und Vielzeller (vgl. Karl Grobben 1908, 492), in Bluttiere und blutlose Tiere (vgl. Wolfgang Kullmann 2014, 137-140) sowie in Wirbeltiere und wirbellose Tiere unterteilen (vgl. Cäsar R. Böttger 1952, 240). Eine differenziertere Systematisierung des Tierreiches kann anhand von Tierstämmen erfolgen. Der Naturforscher Carl von Linné ist ein bekannter Vertreter dieses Einteilungssystems. Er unterteilt das Tierreich in folgende sechs Tierklassen: Säugetiere, Vögel, Amphibien (einschl. Reptilien), Fische, Insekten und Würmer (vgl. Cäsar R. Böttger 1952, 238-240).

\section{Roboter}

Roboter werden für unterschiedliche Bereiche entwickelt und lassen sich dementsprechend kategorisieren (Heidrun Becker 2018, 230). Folgende drei Anwendungsbereiche von Roboter-Tieren sind bisher zentral:

- menschliche Interaktion: sozial-emotionale Roboter-Tiere,

- tierische Interaktion: ultrarealistische Roboter-Tiere (Animaten),

- Cyborgs: Mischwesen.

\section{Menschliche Interaktion (sozial-emotionale Roboter-Tiere)}

Für die menschliche Interaktion wurden bisher verschiedene Roboter-Tiere entwickelt. Es gibt Roboter-Tiere, die als Spielzeug verkauft werden, wie der Roboter-Hund Aibo und Co. (vgl. Florian Rötzer 1999), aber auch welche, die eigens für die menschliche Therapie konstruiert wurden, wie die Roboter-Robbe Paro (vgl. Barbara Klein 2016, 33-34; vgl. Alexander Dietz 2014, 497-499). Spielzeug-Robotertiere sind im Vergleich zu Therapierobotern weniger erforscht. Daher wird im Folgenden exemplarisch Bezug auf Paro genommen.

Die Roboter-Robbe Paro wurde am National Institute of Advanced Industrial Science and Technology in Japan unter der Leitung von Takanori Shibata entwickelt. Paro ist einer Babysattelrobbe nachempfunden und bedient mit ihren Körperproportionen das Kindchen-Schema. Die Robbe ist ca. 57 $\mathrm{cm}$ groß und ca. $3 \mathrm{~kg}$ schwer. Paro wird vor allem in der Demenztherapie und in der tiergestützten Therapie eingesetzt. Sie gehört zu den sozial-emotionalen Robotern und kann mit dem Menschen interagieren sowie nonverbal kommunizieren. Die Robbe ist mit verschiedenen Tast-, Licht-, Akustik-, Lage-, Temperatur- und Spracherkennungssensoren ausgestattet. Der Roboter kann zudem den Kopf und seine Flossen bewegen. Darüber hinaus ist Paro in der Lage, Gefühle mit Hilfe von gezielten Bewegungen und Geräuschen auszudrücken. Die Roboter-Robbe reagiert auf ihren Na- 
men und erinnert sich an frühere soziale Interaktionen. Paro hat ein weiches antibakterielles Fell, welches Risiken hinsichtlich allergischer Reaktionen nahezu ausschließt. Ebenso gering ist die Verletzungswahrscheinlichkeit während der Mensch-Roboter-Interaktion (vgl. Barbara Klein 2016, 33-34; Thomas Hirmann 2015, 8-10 und 15-17; vgl. Alexander Dietz 2014, 496-499).

\section{Tierische Interaktion (ultrarealistische Tierroboter "Animaten“)}

Ultrarealistische Tierroboter werden eigens für die tierische Interaktion konstruiert. Sie sind natürlich entwickelten Tieren olfaktorisch, optisch, haptisch, verhaltens- sowie bewegungstechnisch nachempfunden. Ziel des Einsatzes von Animaten ist es, tiefere Erkenntnisse über das Verhalten sowie Leben der Tiere zu gewinnen (Matthias Haun 22013, 14; Terra X 2018 a; vgl. ZDF 2018 a). Aktuelle Beispiele sind die RoboBee der Freien Universität Berlin von Raúl Rojas und Randolf Menzel (2011), der afrikanische Nilhecht-Roboter-Fisch der Universität Bonn unter der Leitung von Gerhard von der Emde und Martin Worm (Martin Worm et al. 2018) sowie über dreißig verschiedene Wildtier-Animatroniks von John Nolan und seinem Team (Terra X 2018 a; ZDF 2018 a). ${ }^{1}$

\section{Cyborgs (Mischwesen)}

Der Begriff Cyborg umfasst „diejenigen symbiotischen Verbindungen zwischen einem lebenden Organismus und einem künstlichen System, die durch (intraorganistische) Schnittstellen entstehen" (Marion Friedrich 2003, 6). Cyborgs sind demnach vom Menschen optimierte „Mischwesen“, die sowohl natürlich biologische wie auch künstlich konstruierte Elemente innehaben (Marion Friedrich 2003, 5-8). Aktuelle Beispiele sind die Cyborg-Heuschrecke (Coby McDonald 2016) oder der Cyborg-Rochen (Park Sung-Jin et al. 2016, 158-162). So ist die Cyborg-Heuschrecke eine natürlich vorkommende Heuschrecke, die mit einem Chip ausgestattet wird. USForscher haben mit Hilfe von Elektroden, die sie mit dem Gehirn der Heuschrecke verbinden, die Möglichkeit geschaffen, das Gehirn zu „kapern“ und somit den Geruchssinn des Tieres auszulesen und zu nutzen. Zukünftig soll es so möglich sein, den extrem sensiblen Geruchssinn der CyborgHeuschrecke für die Sprengstoffsuche zu nutzen (Coby McDonald 2016).

1 Eine ausführlichere Erörterung der Animaten-Experimente findet im dritten Kapitel statt. 


\section{Gemeinsamkeiten und Unterschiede von natürlich entwickelten Tieren und Roboter-Tieren}

Natürlich entwickelte Tiere werden in der Literatur mit verschiedenen biologischen und psychologischen Eigenschaften beschrieben. Aus biologischer Sicht besitzen natürlich entwickelte Tiere einen Stoffwechsel, können sich selbst reproduzieren, haben eine genetische Variabilität inne und entwickeln sich evolutionär. Darüber hinaus können sie sich bewegen und besitzen Sinnesorgane, die es ihnen ermöglichen, ihre Umwelt wahrzunehmen sowie auf Reize zu reagieren. Aus psychologischer Perspektive sind natürlich entwickelte Tiere empfindungs- und lernfähig, können mit ihrer Umwelt sowohl interagieren als auch kommunizieren und haben ein Erinnerungsvermögen (Reinhard Brandt 2012, 323-334; Georg Töpfer 2011, 494; Dudenredaktion 2019). Je nach Tierklasse sind diese Eigenschaften und Fähigkeiten jedoch unterschiedlich komplex oder primitiv ausgeprägt und entwickelt. Anhand eines Vergleiches werden im Folgenden Gemeinsamkeiten und Unterschiede von natürlich entwickelten Tieren und Roboter-Tieren herausgearbeitet (Übersicht siehe Tabelle 1).

\section{Sozial-emotionale Roboter wie Roboter-Robbe Paro}

Paro ist mit Tast-, Licht-, Akustik-, Temperatur-, Lage- und Spracherkennungssensoren ausgestattet, die es ihm ermöglichen, die Umwelt wahrzunehmen und auf Reize zu reagieren. Der Tierroboter ist darauf programmiert, empfindsam auf Interaktionen zu reagieren und mit Hilfe von Geräuschen und Bewegungen mit seinem Gegenüber zu kommunizieren. So gibt er bei sanften Berührungen fröhliche und bei grobem Kontakt traurige Geräusche von sich und bewegt dementsprechend seinen Kopf, Flossen sowie Augen. Paro ist zudem lernfähig und erinnert sich an frühere Erfahrungen mit seinen Interaktionspartnern. Biologische Eigenschaften und Fähigkeiten hingegen, wie Stoffwechselvorgänge, genetische Variabilität, Selbstreproduktionsfähigkeit und eine evolutionäre Entwicklung, sind bei Paro nicht vorhanden (Barbara Klein 2016, 33-34; Alexander Dietz 2014, 496-499).

\section{Ultrarealistische Tierroboter (Animaten)}

Auch bei den ultrarealistischen Tierrobotern sind aufgrund von verschiedenen Sensoren (Kameras, Mikrofone) Sinnesorgane vorhanden, die es dem Roboter ermöglichen, seine Umwelt wahrzunehmen. Ebenso sind die Tierroboter mit technischen Gelenken ausgestattet, sodass sie sich arttypisch bewegen können. Mit Hilfe von Duftstoffen sowie artspezifischen Geräuschen und Bewegungsmustern ist eine tierische Interaktion möglich. 
Bauelemente, die die Empfindungsfähigkeit, Reizreaktionen und Lernfähigkeit des Roboters ermöglichen, sind je nach Modell unterschiedlich komplex verbaut. Biologische Eigenschaften sind bei den ultrarealistischen Tierrobotern wie bei Paro bisher ebenfalls nicht vorhanden (Martin Worm et al. 2018; Raúl Rojas/Randolf Menzel 2011; Terra X 2018 a; ZDF 2018 a).

\section{Cyborgs (Mischwesen)}

Cyborgs bilden hier eine Ausnahme, da Tiere oder tierische Zellen mit menschlich konstruierten Elementen „verschmelzen“. Je nachdem, wie das Verhältnis der biologisch-tierischen und künstlich-konstruierten Anteile ist, umso mehr bzw. weniger biologische und psychologische Eigenschaften besitzen sie. Die Cyber-Heuschrecke besitzt, da sie ein natürlich entwickeltes Tier ist, welches mit einem Chip versehen ist, alle tierischen Eigenschaften und Fähigkeiten (Coby McDonald 2016).

\begin{tabular}{|l|c|c|c|c|}
\hline & $\begin{array}{c}\text { Natürliche } \\
\text { Tiere }\end{array}$ & Roboter Paro & Animaten & Cyborgs \\
\hline Stoffwechsel & + & - & - & + \\
\hline $\begin{array}{l}\text { Selbst- } \\
\text { reproduktion }\end{array}$ & + & - & - & $-1+$ \\
\hline $\begin{array}{l}\text { Genetische } \\
\text { Variabilität }\end{array}$ & + & - & - & + \\
\hline $\begin{array}{l}\text { Evolutionäre } \\
\text { Entwicklung }\end{array}$ & + & - & - & $-/+$ \\
\hline Sinnesorgane & + & + & + & + \\
\hline $\begin{array}{l}\text { Empfindungen/ } \\
\text { Reizreaktion }\end{array}$ & + & + & $-/+$ & + \\
\hline Bewegung & + & + & + & + \\
\hline Lernfähigkeit & + & + & $-/+$ & $-/+$ \\
\hline $\begin{array}{l}\text { Interaktion/ } \\
\text { Kommunikation }\end{array}$ & + & + & $-/+$ & $-/+$ \\
\hline $\begin{array}{l}\text { Erinnerungs- } \\
\text { vermögen }\end{array}$ & + & + & + & + \\
\hline
\end{tabular}

Tabelle 1: Übersicht: Eigenschafts- und Fähigkeitenvergleich von natürlich entwickelten Tieren und Roboter-Tieren.

Anhand dieser kurzen Eigenschafts- und Fähigkeitsanalyse konnte dargelegt werden, dass natürlich entwickelte Tiere und Roboter-Tiere teilweise ähnliche Eigenschaften und Fähigkeiten aufzeigen, wenngleich auch nicht in identischer Form. 


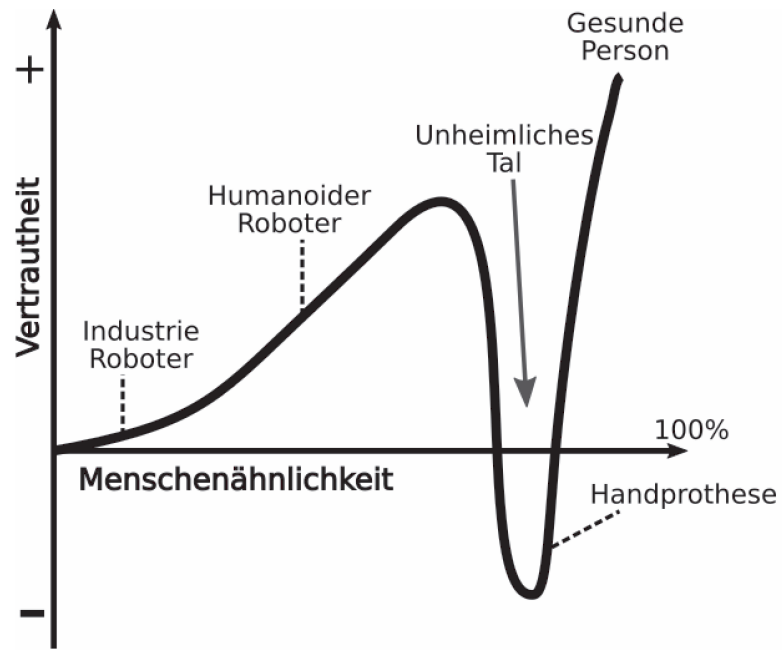

Abb. 1: Das unheimliche Tal (Masahiro Mori 1970).

Wie wirken sich diese ähnlichen Eigenschaften und Fähigkeiten von natürlich entwickelten Tieren und Roboter-Tieren auf Mensch-Robotertierund Tier-Robotertier-Beziehungen aus? Werden Roboter-Tiere von Menschen und Tieren gleichermaßen akzeptiert und als ihresgleichen anerkannt? Oder ist eine Differenz zwischen menschlichem und tierischem Verhalten erkennbar? Diesen Fragen soll in Punkt drei nachgegangen werden.

\section{Anthropomorphismus und "Animorphismus"}

Der Mensch ist ein phantasiereiches Wesen und projiziert von klein an menschliche Eigenschaften auf Entitäten. Je menschenähnlicher Entitäten wie Puppen, Tiere und Roboter aussehen und sich verhalten, umso schneller anthropomorphisiert er sie (Martina Mara/Markus Appel 2017, 3-5). Dieses Phänomen verkehrt sich bei Robotern allerdings ins Gegenteil, sobald der Mensch den Roboter nicht mehr genau einordnen kann. Es kommt zu einem Schubladen-Dilemma (Marcus Cheetham et al. 2011; Christopher H. Ramey 2005; Yuki Yamada et al. 2013). Die Konsequenz: Der Roboter wird als etwas Unheimliches wahrgenommen und der Mensch distanziert sich. Dieser Effekt wird erstmals vom japanischen Robotiker Masahiro Mori beschrieben und als „unheimliches Tal - uncanny valley" bezeichnet (siehe Abb. 1). 
Nach Moris Theorie „steigt mit wachsender Menschenähnlichkeit auch die Vertrautheit mit dem Roboter. Wird er aber zu ähnlich, ruft er Befremden oder gar Angst hervor“ (Jutta Weber 2006, 148). Dieses Dilemma kann vermieden werden, wenn der Roboter entweder eindeutig als solcher zu erkennen ist oder durch seine Menschengleichheit bzw. Tiergleichheit vollständig überzeugen kann (Masahiro Mori 1970, 33-35; Martina Mara/ Markus Appel 2017, 4-5; Ernst Jentsch 1906). So ist die Roboter-Robbe Paro einer Babysattelrobbe nachempfunden. Menschen haben mit Robben in der Regel keine Vorerfahrungen, sodass sie Paro unvoreingenommen begegnen (Takanori Shibata/Kazuo Tanie 2001, 2572-2577; Takanori Shibata et al. 2012, 53-63). Verschiedene Feldstudien, die den Einsatz der Roboter-Robbe erforscht haben, verweisen auf eine hohe Akzeptanz von Paro. Menschen akzeptieren Paro nicht nur ähnlich wie ein lebendiges Tier, sondern sie bevorzugen den Tierroboter teilweise auch als Interaktionspartner. Gründe hierfür waren unter anderem, dass Paro nicht wegläuft, keine Pausen braucht, immer zur Verfügung steht, nicht krank wird oder sterben kann (Hayley Robinson et al. 2013, 664-665; Hayley Robinson et al. 2016; Takanori Shibata et al. 2012, 58-62; Barbara Klein et al. 2013, 89-99). Die Studien legen des Weiteren dar, dass der Einsatz von Paro sowohl positive physische wie auch psychische Effekte auf den Menschen hat. Die Robbe fördert soziale Kontakte, vermindert Stress und Angst, erhöht die Zufriedenheit und wirkt sich somit positiv auf die Atmosphäre und die Vitalwerte von Patienten aus (Takanori Shibata et al. 2012, 58-62; Hayley Robinson et al. 2015; Barbara Klein et al. 2013, 89-99). Darüber hinaus konnte beobachtet werden, dass Menschen Roboter-Tiere anthropomorphisieren. So versuchten Patienten, die Roboter-Robbe Paro zu füttern und sie zuzudecken (Barbara Klein et al. 2013, 95).

Menschen und Tiere sind von klein auf sehr neugierig und zeigen ähnliche Verhaltensweisen. Verhaltensforschungen belegen, dass Menschen und Tiere, insbesondere höherentwickelte Säugetiere, ähnliche Formen des Spielverhaltens aufweisen. Menschen und Tiere üben von klein an das Objektspiel und das soziale Spiel (vgl. Bernd Rosslenbroich 2014, 187, 192). So konnte der Primatologe Richard Wrangham beobachten: „Junge weibliche Schimpansen der Kanyawara-Familiengruppe im Kibale-Nationalpark in Uganda nutzen Stöcke als rudimentäre Puppen und kümmern sich so um sie, wie die Schimpansenmütter der Gruppe sich um ihren echten Nachwuchs kümmern" (Brian Handwerk 2018). Wranghams Beobachtungen belegen, dass junge Schimpansen, ähnlich wie Kinder, mit Stöcken spielen und Gegenständen Eigenschaften zusprechen können. Allerdings wurde „das an Puppenspiel erinnernde Verhalten mit den Stöcken [...] bisher noch nicht bei anderen Populationen beobachtet" (Brian Handwerk 
2018). Trotzdem gibt dieses Verhalten einen ersten Hinweis auf einen "Animorphismus“.

Anhand weiterer Studien und Wildtierdokumentationen soll dargelegt werden, dass Tiere auch Roboter-Tieren arteigene Eigenschaften zuschreiben. Im Folgenden werden Beobachtungen und Studienergebnisse, die weitere Hinweise auf einen „Animorphismus“ geben, aufgezeigt.

Ein Forscherteam der Freien Universität Berlin konstruierte einen ultrarealistischen Bienen-Roboter. Ihnen gelang es, die RoboBee erfolgreich in einen Bienenschwarm zu integrieren. Die natürlich entwickelten Bienen akzeptierten die Roboter-Biene im Bienenvolk und kommunizierten mit ihr erfolgreich mittels Bienentanz. So konnte die RoboBee beispielsweise die Bienen gezielt zu neuen Futterstellen führen (Raúl Rojas/Randolf Menzel 2011).

In einer anderen Studie versuchten Wissenschaftler der Universität Bonn mit Hilfe eines Roboter-Fisches mehr über die artspezifische Kommunikation sowie das Schwarmverhalten von afrikanischen Nilhechten herauszufinden. Sie wollten beweisen, dass Nilhechte mittels elektrischer Signale miteinander kommunizieren. Die These bestätigte sich. Der eingeschleuste Roboter-Fisch wurde erst von den Nilhechten wahrgenommen, als er artspezifische elektrische Signale aussendete. Es konnte beobachtet werden, dass die Nilhechte ihre elektronischen Signale anpassten und dem RoboFisch durch das Becken folgten (vgl. Martin Worm et al. 2018).

Weitere ultrarealistische Tierroboter wurden vom Tierfilmer John Downer und dem Animatronik-Designer John Nolan konstruiert und für Wildtierstudien verwendet. Anhand der Filmaufnahmen eruierten sie, dass Animatroniks „von den Tieren zumindest nicht als störend wahrgenommen, oft sogar mit Interesse untersucht und manchmal anscheinend als Ihresgleichen akzeptiert [wurden]“ (ZDF 2018 a). Bei Animatroniks von afrikanischen Wildhunden, Warzenschweinen, Krokodilen, Schildkröten und den Hanuman-Languren konnte eine hohe Akzeptanz beobachtet werden. So wurden einem afrikanischen Wildhundwelpen-Roboter sowie einem Warzenschwein-Roboter eine arttypische Begrüßung zuteil und sie konnten sich frei im Rudel bzw. in der Horde bewegen, ohne auf Feindseligkeiten zu treffen (Terra X 2018 b; ZDF 2018 a, 2018 b und 2018c). Selbst Zebramangusten ließen sich täuschen und versuchten bei dem Warzenschwein-Animatronik eine Hautpflege durchzuführen. Auch Krokodile akzeptierten den künstlichen Nachwuchs, trugen ihn gemeinsam mit ihrem natürlichen Nachwuchs ins Wasser und beschützten beide vor Fressfeinden (Terra X 2018 b; ZDF 2018 a). Bei den Leopard-Schildkröten konnten Paarungsversuche mit einer Animatronik-Schildkröte beobachtet werden (ZDF 2018 b). Ein eingeschleuster Hanuman-Languren-Baby-Animatronik 
wurde kurzzeitig von einem Langurenweibchen adoptiert. Als sich das Animatronik-Baby allerdings nicht mehr bewegte, hielt sie es für tot und trauerte um das vermeintliche Jungtier (Terra X 2018 a und 2018 b).

All diese Beispiele lassen vermuten, dass Tiere den ultrarealistischen Tierrobotern teilweise ähnliche Bedürfnisse zuschreiben wie ihren natürlichen Artgenossen. Die Eigenschaftsattribution der Wildtiere erfolgte dabei nicht nur im spielerischen Sinne, wie am Beispiel der Schimpansen ersichtlich wurde. Einige Wildtiere erkannten die ultrarealistischen Tierroboter als ihresgleichen an, was anhand der aufgezeigten Tier-Roboter-Interaktionen deutlich wurde.

Im Gegensatz zum Menschen scheint es bei den Tieren kein „unheimliches Tal - uncanny valley“ zu geben. Entweder konnte der ultrarealistische Tierroboter olfaktorisch, optisch, haptisch, verhaltens- sowie bewegungstechnisch überzeugen und wurde im Wildtierverbund aufgenommen oder er wurde kurzzeitig begutachtet, zurückgelassen oder gar zerstört (Raúl Rojas/Randolf Menzel 2011; Martin Worm et al. 2018; ZDF 2018 a und $2018 \mathrm{c}$ ).

Die Analyse legt somit nahe, dass es auch im Tierreich eine Art „Animorphismus" gibt. Jedoch ist unklar, inwieweit es den Wildtieren bewusst war, dass es sich bei den Roboter-Tieren um keine „natürlichen“ Interaktionspartner handelte.

\section{Tierethische Ansätze in Bezug auf Roboter-Tiere}

Aufgrund der bisher aufgezeigten Ähnlichkeiten, der Akzeptanz und der vielfältigen Einsatzfelder von Roboter-Tieren sowohl in der menschlichen wie auch in der tierischen Lebenswelt werden im Folgenden exemplarisch zwei tierethische Ansätze auf Roboter-Tiere angewandt. Anhand des „Verrohungsarguments“ von Immanuel Kant und des Tierrechtsansatzes von Tom Regan soll untersucht werden, inwieweit nicht nur natürlich vorkommenden Tieren, sondern auch Roboter-Tieren eine Art moralischer Status gebührt und ihnen ein inhärenter Wert zukommen sollte.

\section{1 „Verrohungsargument" von Immanuel Kant}

Das „Verrohungsargument“ besagt, dass „der Mensch Tiere human behandeln sollte, und zwar deshalb, um nicht zu verrohen und schlussendlich Gefahr zu laufen, auch Menschen gegenüber grausam und rücksichtslos zu 
werden“ (Martin M. Lintner 2017, 72; siehe auch Heike Baranzke 2018; Immanuel Kant 1907, $\mathbb{1} 17)$. Ausgehend von der eben dargelegten kongruenten menschlichen Akzeptanz und dem Anthropomorphisieren von natürlich vorkommenden Tieren und Roboter-Tieren kann das „Verrohungsargument" auch auf Roboter-Tiere übertragen werden. Wenn der Mensch Roboter-Tieren ähnliche Eigenschaften zuschreibt wie sich selbst, läuft er Gefahr, moralisch abzustumpfen, wenn er Roboter-Tiere - gleichermaßen wie lebendige Tiere - schlecht behandelt. Auch wenn Kant, wie häufig kritisiert, anthropozentrisch argumentiert, ist es ein plausibles Argument für Verhaltensregeln sowohl gegenüber natürlich vorkommenden Tieren wie auch gegenüber Roboter-Tieren.

\subsection{Tierrechtsansatz von Tom Regan}

Tom Regan plädiert in seinem Tierrechtsansatz für einen respektvollen Umgang innerhalb einer moralischen Gemeinschaft. Jedes Mitglied einer moralischen Gemeinschaft besitzt einen inhärenten Wert und hat gleichermaßen das Recht auf Leben, körperliche Unversehrtheit und Freiheit. Zur moralischen Gemeinschaft gehören für ihn alle Lebewesen, die Subjekt-eines-Lebens sein können. Nach Regan sind dies nicht nur Menschen, sondern auch Tiere, insbesondere Säugetiere und Vögel, da sie mit dem Menschen nicht nur biologisch und psychologisch verwandt sind, sondern auch der Welt gewahr sind. Die biologische und psychologische Verwandtschaft zwischen Menschen und Tieren ist nach Regan an folgenden Merkmalen erkennbar (Tom Regan 2014, 100-114):

- gleiche Sprache und Verhalten in Bezug auf nonverbale Kommunikation und Interaktion,

- gleicher Körper hinsichtlich der Sinnes- und Körperorgane,

- gleiches System bezüglich des Nervensystems, welches Signale an das Gehirn weiterleitet, um beispielsweise Verletzungen zu vermeiden,

- gleicher Ursprung: göttliche Schöpfung oder evolutionäre Entwicklung.

Menschen und natürlich entwickelte Tiere sind sich, wie Regan in seinem Tierrechtsansatz deutlich macht, sehr ähnlich und zeigen viele psychische und physische Gemeinsamkeiten auf (Tom Regan 2014, 102-110). RoboterTiere werden nach tierischen und menschlichen Vorbildern erbaut. Wenn es Ähnlichkeiten zwischen Menschen und natürlich entwickelten Tieren gibt, dann gibt es diese auch bei Roboter-Tieren. Um dieser These nachzugehen, soll im Folgenden untersucht werden, wie Regans Merkmale auf Roboter-Tiere anzuwenden sind. 


\section{Sprache und Verhalten}

Es kann festgestellt werden, dass Roboter-Tiere ähnlich dem Menschen und natürlich entwickelten Tieren sowohl eine Sprache als auch ein Verhalten innehaben. Je nach Einsatzgebiet bekommen Roboter-Tiere eine arttypische Sprache und Verhaltensweisen einprogrammiert. Eigene Bedürfnisse und Ängste können Roboter-Tiere zwar vortäuschen, diese sind jedoch nicht echt. Erinnerungen hingegen sind, wie bei Paro aufgezeigt, heute schon Realität.

\section{Gleicher Körper}

Roboter-Tiere weisen ähnliche Sinnesorgane auf, mit denen sie ihre Umwelt wahrnehmen und auf Reize reagieren können. Sie haben mehrere mechanische Gelenke, die es ihnen ermöglichen, sich arttypisch zu bewegen. Lebensnotwendige Organe aus Zellen wie Herz und Lunge sind nicht vorhanden. Ausnahmen bilden diesbezüglich Cyborgs.

\section{Gleiches System}

Roboter-Tiere haben eine Art Nervensystem in Form von Sensoren, die alle Informationen in einem zentralen Rechner („Roboter-Gehirn“) verarbeiten und angenehme wie auch unangenehme Interaktionen wahrnehmbar machen.

\section{Gleicher Ursprung}

Ein gleicher Ursprung ist im direkten Sinne nicht vorhanden. Indirekt könnte man von einer „Evolution 2.0“ oder „Schöpfung 2.0“ sprechen, da der Mensch Roboter-Tiere aus Materialien, die er in der Welt vorfindet, konstruiert und immer weiter optimiert.

\section{Subjekt-eines-Lebens}

Neben der physischen und psychologischen Verwandtschaft betont Regan allerdings noch einen weiteren Aspekt, der ein Lebewesen zu einem Subjekt-eines-Lebens macht. Für Regan sind alle Lebewesen Subjekte-eines-Lebens, die der Welt gewahr sind. Das bedeutet: Subjekte-eines-Lebens merken stets, was mit ihnen geschieht, da es sich auf ihre Lebensqualität und Dauer auswirkt (Tom Regan 2014, 100-103). Das ist auch „unabhängig davon, ob sich jemand anderes dafür interessiert oder nicht" (Tom Regan 2014, 101). Dies ist ein entscheidender Unterschied zwischen natürlich entwickelten Lebewesen und Robotern. Für Roboter-Tiere ist es bisher noch nicht von Bedeutung, was mit ihnen passiert. Roboter-Tiere sind somit nach Regan zum heutigen Stand der Technik keine Subjekte-eines-Lebens. Aufgrund ihrer täuschend echten Erscheinung neigen Mensch und 
Tier jedoch dazu, den Roboter-Tieren Attribute wie Schmerz, Angst und Freude zuzuschreiben, auch wenn Roboter diese nicht innehaben.

\section{Fazit}

Roboter-Tiere sind auf der einen Seite insofern ein verheißungsvolles Experiment, da sowohl Menschen als auch natürlich entwickelte Tiere RoboterTiere häufig akzeptieren und mit ihnen erfolgreich interagieren. Darüber hinaus können Roboter-Tiere das menschliche und tierische Leben bereichern, da sie als Interaktionspartner Einsamkeitsgefühle verringern und das Sozialverhalten verbessern können. Ein Verletzungsrisiko und die Gefahr von Allergien sind so gut wie ausgeschlossen.

Auf der anderen Seite sind Roboter-Tiere hinsichtlich fehlender natürlich-biologischer Eigenschaften und den stark beschränkten Interaktionsmöglichkeiten noch nicht ausgereift.

Wie die Roboter-Tier-Analyse gezeigt hat, sprechen Menschen und natürlich entwickelte Tiere Roboter-Tieren trotz technischer Unvollständigkeit arttypische Eigenschaften zu. Ausgehend von den tierethischen Ansätzen spricht aus anthropozentrischer Sicht einiges dafür, Roboter-Tieren einen niederen moralischen Status zukommen zu lassen. Der Mensch sollte sich, im Sinne von Kant, auch im Umgang mit Roboter-Tieren vor einer Verrohung schützen.

Nach Regans Tierrechtsansatz gebührt Roboter-Tieren zum heutigen Stand der Technik aus pathozentrischer Perspektive kein moralischer Status, da Roboter-Tiere keine Subjekte-eines-Lebens sind; auch wenn Roboter-Tiere viele ähnliche Merkmale zu natürlich entwickelten Tieren aufweisen.

Die in dieser Arbeit untersuchten Forschungs- und Einsatzfelder legen nahe, dass Roboter-Tiere nicht ausschließlich ein Themenbereich der Maschinenethik sind, sondern auch im tierethischen Diskurs berücksichtigt werden müssen. Roboter-Tiere werden in Zukunft in der menschlichen und in der tierischen Lebenswelt vermehrt Einzug halten. Es wird häufiger zu Begegnungen zwischen Menschen, natürlich entwickelten Tieren und Roboter-Tieren kommen. Es müssen u. a. folgende Fragen geklärt werden: Ist die identische Nachkonstruktion von Lebewesen moralisch vertretbar? Wenn ja, inwiefern sollte den Tierrobotern zukünftig ein moralischer Status zustehen? Inwieweit dürfen Roboter-Tiere und Cyborgs entwickelt und für menschliche Zwecke instrumentalisiert werden? Verändern RoboterTiere die menschliche Sicht auf natürlich entwickelte Tiere? Und welchen 
Einfluss nehmen ultrarealistische Tierroboter und Cyborgs auf die natürliche Tierwelt?

\section{Literatur}

Baranzke, Heike 2018, Verrohungsargument, in: Ach, Johann S./Borchers, Dagmar (Hg.) 2018, Handbuch Tierethik, Stuttgart, 219-224.

Becker, Heidrun 2018, Robotik in der Gesundheitsversorgung: Hoffnungen, Befürchtungen und Akzeptanz aus Sicht der Nutzerinnen und Nutzer, in: Bendel, Oliver (Hg.) 2018, Pflegeroboter, Wiesbaden, 229-248.

Böttger, Cäsar R. 1952, Die Stämme des Tierreichs in ihrer systematischen Gliederung (Abhandlungen der Braunschweigischen Wissenschaftlichen Gesellschaft 4), Braunschweig, 238-300.

Brandt, Reinhard 2012, Wahrnehmen, Fühlen, Verhalten, Denken: Was können Tiere, in: Deutsche Zeitschrift für Philosophie 60 (3), 323-334.

Cheetham, Marcus/Suter, Pascal/Jäncke, Lutz 2011, The human likeness dimension of the „uncanny valley hypothesis“: behavioral and functional MRI findings, in: Frontiers in Human Neuroscience 5, art. 126.

Dietz, Alexander 2014, Roboter in der Altenpflege?, in: Pastoraltheologie 103, 496-517.

Dudenredaktion 2019, Art. Tiere, in: https://www.duden.de/rechtschreibung/Tier (abgerufen am 04.09.2019).

Friedrich, Marion 2003, Die künstliche Evolution der Cyborgs. Erkenntnistheoretische Aspekte der Bioinformatik (diplomica 5), Marburg.

Grobben, Karl 1908, Die systematische Einteilung des Tierreiches, in: Verhandlungen der Zoologisch-Botanischen Gesellschaft 58, Wien, 491-511.

Handwerk, Brian 2018, Weibliche Schimpansen spielen mit „Puppen“, in: National Geographic, online: https://www.nationalgeographic.de/tiere/2018/09/weibli che-schimpansen-spielen-mit-puppen (abgerufen am 18.01.2020).

Haun, Matthias ${ }^{2} 2013$, Handbuch Robotik. Programmieren und Einsatz intelligenter Roboter, Berlin/Heidelberg.

Hirmann, Thomas 2015, Die Möglichkeiten und Auswirkungen von sozial-emotionalen Robotern, insbesondere der Robbe Paro, im Einsatz in der Pflege, Wien.

Jentsch, Ernst 1906, Zur Psychologie des Unheimlichen, in: Psychiatrisch-neurologische Wochenschrift 22/23, 195-198, 203-205.

Kant, Immanuel 1907, Die Metaphysik der Sitten (Kants Werke. Akademie-Textausgabe VI), Berlin.

Klein, Barbara 2016, Zwischen Natur und Technik - Künstliche Tiere. Können künstliche Tiere zur Lebensqualität in der Altenpflege beitragen, in: Fehlmann, Meret/Michel, Margot/Niederhauser, Rebecca (Hg.) 2016, Tierisch! Das Tier und die Wissenschaft: Ein Streifzug durch die Disziplinen, Zürich, 33-42. 
Klein, Barbara/Gaedt, Lone/Cook, Glenda 2013, Emotional Robots - Principles and Experiences with Paro in Denmark, Germany and the UK, in: The Journal of Gerontopsychology and Geriatric Psychiatry 6, 89-99.

Kullmann, Wolfgang 2014, Aristoteles als Naturwissenschaftler, Göttingen.

Lintner, Martin M. 2017, Der Mensch und das liebe Vieh. Ethische Fragen im Umgang mit Tieren. Mit Beiträgen von Christoph J. Amor und Markus Moling, Innsbruck.

Mara, Martina/Appel, Markus 2017, Roboter im Gruselgraben: Warum uns menschenähnliche Maschinen oft unheimlich sind, Linz.

McDonald, Coby 2016, Brain-Jacked Locusts Could Be The Next Bomb Detectors. Turning a much maligned insect into a biorobotic hero, in: Popular Science, Technology, in: https://www.popsci.com/navy-funds-research-into-cyborg-locusts/ (abgerufen am 20.01.2020).

Mori, Masahiro 1970, Bukimi no tani [The uncanny valley], in: Energy 7, 33-35.

Ramey, Christopher H. 2005, The uncanny valley of similarities concerning abortion, baldness, heaps of sand, and humanlike robots, in: Proceedings of Views of the Uncanny Valley Workshop: IEEE-RAS International Conference on Humanoid Robots, Tsukuba, Japan, 8-13.

Regan, Tom 2014, Von Menschenrechten zu Tierrechten (aus dem Englischen übersetzt von Matthias Kiesselbach und Friederike Schmitz), in: Schmitz, Friederike (Hg.) 2014, Tierethik. Grundlagentexte, Berlin, 102-110.

Robinson, Hayley/MacDonald, Bruce/Kerse, Ngaire/Broadbent, Elizabeth 2013, The Psychosocial Effects of a Companion Robot: A Randomized Controlled Trial, in: Journal of the American Medical Directors Association 14 (9), 661-667.

Robinson, Hayley/MacDonald, Bruce/Broadbent, Elizabeth 2015, Physiological effects of a companion robot on blood pressure of older people in residential care facility: A pilot study, in: Australasian Journal on Ageing 34 (1), 27-32.

Robinson, Hayley/Broadbent, Elizabeth/MacDonald, Bruce 2016, Group sessions with Paro in a nursing home: Structure, observations and interviews, in: Australasian Journal on Ageing 35 (2), 106-112.

Rojas, Raúl/Menzel, Randolf 2011, RoboBee: Ein biomimetischer Bienenroboter zur Erforschung des Bienentanzes, in: https:/www.dfg.de/dfg_magazin/veransta ltungen/ausstellungen/idee_erkenntnis/robobee/ index.html (abgerufen am 28.08.2019).

Rosslenbroich, Bernd 2014, Die evolutionäre Bedeutung des Spiels, in: Jahrbuch für Goetheanismus 31, 185-217.

Rötzer, Florian 1999, Aibo - der Roboterhund. Noch ein intelligentes Spielzeug, Telepolis, in: https://www.heise.de/tp/features/Aibo-der-Roboterhund-3446466.h tml (abgerufen am 30.01.2020).

Shibata, Takanori/Tanie, Kazuo 2001, Physical and affective interaction between human and mental commit robot, in: Proceedings ICRA. IEEE International Conference on Robotics and Automation (Cat. No.01CH37164), Seoul, 2572-2577. 
Shibata, Takanori/Kawaguchi, Yukitaka/Wada, Kazuyoshi 2012, Investigation on People Living with Seal Robot at Home, in: International Journal of Social Robotics 4, 53-63.

Sung-Jin, Park et al. 2016, Phototactic guidance of a tissue-engineered soft-robotic ray, in: Science, Vol. 353, Issue 6295, 158-162.

Terra X 2018 a, Spione im Tierreich. Große Gefühle, 2018, in: https:/www.fernsehs erien.de/terra-X/folgen/spione-im-tierreich-grosse-gefuehle-1156038 (abgerufen am 27.08.2019).

Terra X 2018 b, Spione im Tierreich. Große Gefühle, 2018, in: https:/www.dailym otion.com/video/x6dguox (abgerufen am 30.01.2020).

Töpfer, Georg 2011, Art. Tier, in: Töpfer, Georg 2011, Historisches Wörterbuch der Biologie. Geschichte und Theorie der biologischen Grundbegriffe, Band 3: Parasitismus - Zweckmäßigkeit, Berlin/Heidelberg, 494-509.

Weber, Jutta 2006, Der Roboter als Menschenfreund. Wie das neue Forschungsfeld Mensch-Roboter-Interaktion den Dienstleistungsbereich erobern will, in: c't Magazin für Computertechnik 2006, Heft 2, 144-149.

Worm, Martin et al. 2018, Evidence for mutual allocation of social attention through interactive signaling in a mormyrid weakly electric fish, in: Proceedings of the National Academy of Sciences USA (PNAS) 115 (26), 6852-6857; DOI: 10.1073/pnas.1801283115.

Yamada, Yuki/Kawabe, Takahiro/Ihaya, Keiko 2013, Categorization difficulty is associated with negative evaluation in the "uncanny valley" phenomenon, in: Japanese Psychological Research 55, 20-32.

ZDF 2018 a, Animatroniks im Einsatz, in: https://www.zdf.de/dokumentation/ terra-x/spione-im-tierreich-animatroniks-im-einsatz-100.html (abgerufen am 04.09.2019).

ZDF 2018 b, Animatronik trifft echtes Tier, in: https:/www.zdf.de/dokumentation/ terra-x/animatronik-trifft-echtes-tier-100.html (abgerufen am 20.08.2019).

ZDF 2018 c, Making of. Spione im Tierreich. Orang-Utan- und Schimpanse-Animatronik, in: https://www.zdf.de/dokumentation/terra-x/making-of-spione-im-ti erreich-orang-utan-schimpanse-animatronik-100.html (abgerufen am 29.09.2019). 
